\title{
Structural and Electronic Properties of Lead Sulfide Quantum Dots from Screened Hybrid Density Functional Calculations including Spin-Orbit Coupling Effects
}

\author{
Antonio M. Márquez*, Laura C. Pacheco and Javier Fdez. Sanz \\ Departamento de Química Física, Facultad de Química, Universidad de Sevilla, \\ 41012 Sevilla, Spain
}

*Corresponding author. Email: marquez@us.es

\begin{abstract}
We present in this work density functional theory calculations of the structural and electronic properties of $(\mathrm{PbS})_{\mathrm{n}}$ nanoparticles with $\mathrm{n}=4-32$. Particular care has been taken on the correct description of their electronic structure by using a hybrid functional including the spin-orbit coupling effects. We demonstrate that the bonding in $\mathrm{PbS}$ nanoparticles is quite different from bulk $\mathrm{PbS}$ as the six $\mathrm{Pb}-\mathrm{S}$ bonds around a single $\mathrm{Pb}$ atom are found to have a different character while in bulk $\mathrm{PbS}$ all $\mathrm{Pb}-\mathrm{S}$ distances are equivalent. We also relate the geometric structure to the evolution of the HOMOLUMO gaps and show how the computed electronic properties strongly depend on the functional used. Finally, based on an extrapolation of our results we predict that to obtain the onset of absorption in the infrared region of the spectrum a cubic nanoparticle of $\sim 1.96 \mathrm{~nm}$ side length is needed, a result that is in agreement with existing experimental information.
\end{abstract}

Keywords: Lead sulfide, PbS nanoparticles, DFT, spin-orbit, structure, absorption spectrum. 


\section{Introduction.}

Lead chalcogenides quantum dots (QDs) have been extensively studied over the years in relation to their unique chemical, structural and electronic properties compared to their bulk counterparts. The tunability of their electronic properties, related to strong quantum confinement effects arising from the restriction of the electronic wave function to limited regions of space, results in size-dependent electronic properties (see [1] and references therein). The possibility of controlling these properties makes lead chalcogenides interesting candidates for many applications, including energy storage and conversion [2,3], optoelectronics [4], sensing [5,6], and photocatalysis [7].

In particular, lead sulfide (PbS) quantum dots have attracted much attention in recent years in relation both to the favorable properties of bulk PbS (large Bohr radius of $18 \mathrm{~nm}$, narrow band gap of $0.14 \mathrm{eV}$ and high carrier mobility), the availability of synthetic routes for creating size tuned PbS QDs [8], and to their higher stability in comparison to PbSe QDs [9,10]. For these reasons, the structures and electronic properties of $\mathrm{PbS}$ nanoparticles have been examined, both theoretically and experimentally. However, even the most recent density functional theory calculations have been limited by the use of either generalized gradient approximation (GGA) based functionals [11], that are known to underestimate the band gaps due to the selfinteraction error, or by the use of the hybrid B3LYP exchange-correlation functional $[12,13]$, known to largely overestimate the band gaps and to fail in the description of metallic systems $[14,15]$. Moreover, the accurate description of the electronic structure of these systems requires taking into account the semicore $\mathrm{Pb} \mathrm{d}$ states and the incorporation of the relativistic effects (spin-orbit coupling, SOC) for the valence $\mathrm{Pb}$ electrons [16]. Finally, these studies have been limited to compute the HOMO-LUMO gaps and, to the best of our knowledge, only one work has examined, through ab initio CID calculations, the electronic excitation spectrum of some $(\mathrm{PbS})_{\mathrm{n}}$ nanoparticles [12].

Here we present, for the first time, accurate DFT calculations based on the screened hybrid Heyd-Scuseria-Ernzerhoff functional including the spin-orbit coupling effects, known to significantly improve the description of bulk $\mathrm{Pb}$ chalcogenides with respect to standard DFT-GGA functionals, in particular electronic properties like the order of band gaps and effective carrier masses. We explore geometric parameters, bonding energies and electronic structures (HOMO-LUMO gaps and electronic absorption spectra) of $(\mathrm{PbS})_{n}$ QDs with $n$ ranging from $n=4$ to $n=32$. Our results allow 
us to demonstrate that the bonding in $(\mathrm{PbS})_{\mathrm{n}}$ QDs is quite different from bulk PbS as the six $\mathrm{Pb}-\mathrm{S}$ distances around a single $\mathrm{Pb}$ atom are found to have different character while in bulk $\mathrm{PbS}$ all distances are equivalent. We also show how the description of the electronic properties on these systems (HOMO-LUMO gaps and electronic absorption spectra) strongly depends on the functional used. Based on an extrapolation of our

hybrid density functional calculations with SOC effects we predict that to have a HOMO-LUMO gap in the IR region of the spectrum $(1.65 \mathrm{eV})$ a cubic NP of at least $1.96 \mathrm{~nm}$ side length will be required, a result which is in agreement with the experimental observation of band gap of $1.5 \mathrm{eV}$ for PbS QDs of $2.6 \mathrm{~nm}$ diameter.

\section{Computational details.}

The initial geometries of $(\mathrm{PbS})_{\mathrm{n}}$ clusters $(\mathrm{n}=4-32)$ were constructed taking as starting point the cubic arrangement of $(\mathrm{PbS})_{4}$. Larger clusters were built by adding $\mathrm{PbS}$ units to the lower sized clusters and, generally, maintaining the orthorhombic structure except in $(\mathrm{PbS})_{5}$ where a lower symmetry structure was found and in $(\mathrm{PbS})_{6}$ where an isomer with a $\mathrm{C}_{6}$ symmetry axis was also found. The optimized geometries of $(\mathrm{PbS})_{\mathrm{n}}$ nanoparticles (NP) are depicted in Figure 1.

Total energy calculations were carried out using the VASP 5.4 code $[17,18,19]$ with the projector-augmented wave method (PAW) [20,21]. In these calculations, the energy was computed using the generalized gradient approximation (GGA) of DFT as proposed by Perdew, Burke, and Ernzerhof (PBE) [22]. However, standard GGA methods are known to produce too small band gaps due to the incomplete cancellation of the Coulomb self-interaction. For this reason, the screened hybrid functional proposed by Heyd et al. [23] (HSE06 hereafter referred to as HSE), as implemented in the VASP code, that is known to perform extremely well for lead chalcogenides [16] was also used through this work. As the band gaps of bulk lead chalcogenides have been found to be strongly influenced by the magnitude of the spin-orbit coupling of the $\mathrm{Pb} 6 p$ states their effects on the geometrical and electronic structures of $(\mathrm{PbS})_{\mathrm{n}} \mathrm{NPs}$ was also evaluated. In all these calculations electronic states were expanded using plane wave basis sets with a cutoff of $400 \mathrm{eV}$. Forces on the ions were calculated through the Hellman-Feynman theorem, including the Harris-Foulkes correction to forces [24]. Iterative relaxation of the atomic positions was stopped when the forces on the atoms 
were $<0.01 \mathrm{eV} / \AA$. The $(\mathrm{PbS})_{\mathrm{n}}$ NPs where situated in an orthorhombic cell with a vacuum width of at least $15 \AA$ on each direction. Given the size of these cells all calculations were performed at the $\Gamma$ point of the Brillouin zone. The average $\mathrm{Pb}-\mathrm{S}$ bond energy of all $(\mathrm{PbS})_{\mathrm{n}} \mathrm{NP}$ where computed as

$$
E_{B}=\left[E_{N P}-n E_{P b}-n E_{S}\right] / n_{\text {bonds }}
$$

where $E_{P b}$ and $E_{S}$ are the corresponding atomic energies, and $n_{b o n d s}$ is the number of $\mathrm{Pb}-\mathrm{S}$ bonds on the NP.

\section{Results and discussion.}

Figure 2 shows the average $\mathrm{Pb}-\mathrm{S}$ distances for the selected $(\mathrm{PbS})_{\mathrm{n}}$ NPs obtained with the different density functionals employed in this study. There is a clear increase in the average $\mathrm{Pb}-\mathrm{S}$ bond length with increasing $\mathrm{NP}$ size from $(\mathrm{PbS})_{4}$ to $(\mathrm{PbS})_{32}$ in all cases. The inclusion of the computationally expensive spin-orbit coupling terms into the calculations shows to have a negligible effect on the computed Pb-S distances. In no case there is a variation larger than $0.005 \AA$ (PBE) or $0.012 \AA$ (HSE) related to the inclusion of the SOC effects. The trend is the same whether the pure GGA PBE or the hybrid HSE functional are employed to describe the electronic structure of these systems. The only apparent effect is a small shortening of about $0.02 \AA$ in the HSE average $\mathrm{Pb}-\mathrm{S}$ distances compared with the PBE average distances. This shortening is practically identical to the effect found in another study of bulk lead chalcogenides that reports Pb-S distances of $3.002 \AA$ (PBE) and $2.9815 \AA$ (HSE03) [16]. As stated previously, the average $\mathrm{Pb}-\mathrm{S}$ distances increase progressively with increasing NP size, however a significant variation in individual $\mathrm{Pb}-\mathrm{S}$ distances within each cluster is found. A closer look at the origin of this behavior shows that it is related to the geometry of the NPs. At corner sites Pb-S distances ( 2.72 $\AA$ at PBE) are shorter than at the edges ( 2.86 $\AA$ ), and these are shorter than for hexacoordinated sites ( 3.0 $\AA$ ). Thus, for smaller NPs the average bond distances are shorter, as the proportion of corner and edge sites is higher. As the NP size increases, the proportion hexacoordinated sites increase and, thus, the average $\mathrm{Pb}-\mathrm{S}$ distance approach that of bulk PbS. However, a detailed examination of the geometries of individual NPs reveals that not all edges or hexacoordinated sites distances are equal. Broadly speaking tricoordinated $\mathrm{Pb}$ atoms have two shorter bonds, aprox. $2.68 \AA$ (PBE) and another bond slightly elongated, 
$\sim 2.79 \AA$, while in tetracoordinated $\mathrm{Pb}$ atoms the shorter bond is $\sim 2.74 \AA$, there are two longer bonds at $\sim 2.81 \AA$, and a much longer bond at aprox. $3.2 \AA$. This behavior has been observed in some lead chalcogenide complexes [25], alkylxanthates, and diethyldithiophosphates [26]. The elongation of the central $\mathrm{Pb}-\mathrm{S}$ bond in $(\mathrm{PbS})_{8}$ was already noted in a previous study that used the B3LYP functional [12]. However, there are no references to the observation of the same kind of distortion on higher sizes NP and there are no details about the remaining Pb-S distantes. Similarly, Franceschetti, in its study of PbSe nanoparticles [27] found Pb-Se distances significantly distorted with respect to bulk PbSe, finding a relation to the distance to the nanocrystal surface. However, he used quasi-spherical shape nanoparticles, with significant amounts of lowstability (111) faces present, a factor that may have an important influence in the structural parameters of these NPs.

These distances show that the NPs (except those that show non-cubic geometries) seem to be composed of a collection of $(\mathrm{PbS})_{4}$ units. That is, the higher size NPs are built in from the addition of a basic unit: the $(\mathrm{PbS})_{4} \mathrm{NP}$. That must reflect some intrinsic stability of this basic unit. The average $\mathrm{Pb}-\mathrm{S}$ bond energies plotted in Figure 3 effectively show a decrease in bond energy with increasing NP size. The bond energy is higher for $(\mathrm{PbS})_{4}, 2.77 \mathrm{eV}$ (PBE) or $3.31 \mathrm{eV}$ (HSE) and decreases up to $2.0 \mathrm{eV}$ (PBE) or $2.32 \mathrm{eV}$ (HSE) for the larger NP, $(\mathrm{PbS})_{32}$. Previous studies have reported a monotonic increase in binding energies for $\mathrm{n}<5$ and an even-odd oscillation for increasing NP size. This behavior, that seems to be contradictory with our present results, is related to the fact that what these authors present as binding energies are either atomization energies [12] or the average energy needed to decompose the NP into PbS units. [13] The continuous decrease that we observe in the average bond energy can be explained by the higher number of long (thus weaker) Pb-S bonds that appear as the $\mathrm{NP}$ size increase. In fact, the $(\mathrm{PbS})_{8} \mathrm{NP}$ can be viewed as the interaction of two $(\mathrm{PbS})_{4}$ units and, thus, the bond energy of one of those longer bonds can be computed as

$$
\Delta E_{\text {bond }}=\left(E_{(P b S)_{8}}-2 E_{(P b S)_{4}}\right) / 4
$$

since there are four of these bonds in the $(\mathrm{PbS})_{8}$ octamer. From our data, this bond energy can be estimated to be $0.144 \mathrm{eV}(0.136 \mathrm{eV})$ when the PBE (HSE) functional is employed. Similar values are obtained in the larger size NP studied in this work, $(\mathrm{PbS})_{32}$, that looks (regarding the Pb-S distances) like a collection of 8 of those weakly 
interacting $(\mathrm{PbS})_{4}$ units. This means that these longer bonds are intrinsically weak. The reason behind this behavior can be traced to the covalent nature of the $\mathrm{PbS}$ bonding in these NP in contraposition to the metallic or extended nature of the bond in bulk PbS. In fact, no distortion in the cubic structure is observed in bulk PbS. Furthermore, the optimization of a lineal infinite $(\mathrm{PbS})_{\mathrm{n}}$ system (with $\mathrm{PBC}$ conditions) shows that the geometrical distortion observed in the NP disappear (i.e. all Pb-S distances in the axis direction are identical in this extended system) as the bonding becomes expanded at least in one dimension. This result probably implies that as the NP size increases the inner part of the NP will become more "metallic" while it is logical to assume that the external part will preserve the covalency of the Pb-S bond.

The HOMO-LUMO gaps, presented in Figure 4, show a general decreasing trend with NP size in agreement with previous studies of other semiconductor NPs. The HSE gaps are higher than those calculated with the PBE functional by 0.7-0.8 eV but the trend is the same. The inclusion of the SOC effects reduces the gap by $0.5-0.8 \mathrm{eV}$ not only because they split the $\mathrm{Pb}$ levels but also because they change the character of the PbS bond. While the atoms-projected density of states (DOS) shows that either at the PBE or HSE functionals the occupied levels near the HOMO have similar contributions from both atoms, the inclusion of the SOC effects results in an increased ionicity, with higher energy occupied levels constructed mainly from S levels and lower unoccupied orbitals showing higher $\mathrm{Pb}$ contributions.

In both cases (PBE and HSE functionals) there is, for a given NP size, some variations related to the NP geometry. This is quite evident for the $(\mathrm{PbS})_{6},(\mathrm{PbS})_{10}$, and $(\mathrm{PbS})_{16}$ NPs. This behavior can be related to the NP shape, stability, and number of shorter bonds in the structure. Generally speaking, cubic shape NPs are always more stable than non-cubic ones due to the formation of stronger Pb-S bonds. In consequence structures with weaker and, thus, larger bonds are of lower stability and show a smaller HOMO-LUMO gap. Of the two $(\mathrm{PbS})_{6} \mathrm{NPs}$, structure 6b is more stable by $0.09 \mathrm{eV}$ when the PBE functional is used ( $0.15 \mathrm{eV}$ with the HSE functional) and shows a smaller gap of $2.03 \mathrm{eV}(2.85 \mathrm{eV})$ against $2.65 \mathrm{eV}(3.50 \mathrm{eV})$ for structure $6 \mathrm{a}$, that shows hexagonal symmetry. However, in the cubic structure there are only 12 short distances (less than $2.8 \AA$ ) while there are 18 short distances in structure 6a, but the longer distance in structure $6 \mathrm{a}$ is $2.91 \AA$ while in the cubic structure $6 \mathrm{~b}$ the longer distance is only $2.70 \AA$, explaining the lower gap found in this case. The $(\mathrm{PbS})_{10}$ structures are all 
cubic, e.g. can be constructed by joining together or fusing various $(\mathrm{PbS})_{4}$ units. The spread of the gap is, now, directly related to the number of shorter bonds. Structures $10 \mathrm{~d}$ and 10e that present the smaller number of such shorter bonds, have the lower gaps, $1.78 \mathrm{eV}$ and $1.88 \mathrm{eV}$ (2.44 eV and $2.59 \mathrm{eV}$ at HSE functional). (PbS) ${ }_{16}$ structures are, again, all derived from $(\mathrm{PbS})_{4}$ units and their HOMO-LUMO gaps show a similar behavior than for $(\mathrm{PbS})_{10}$ NPs. The lower energy structure, 16a, is composed of four $(\mathrm{PbS})_{4}$ units in a plane. Structure $16 \mathrm{~b}$ is a tridimensional cross-bar structure that results of fusing these four $(\mathrm{PbS})_{4}$ units in a 3D structure, but the units are strongly distorted and the structure is the less stable by $0.61 \mathrm{eV}$ (PBE) or $0.58 \mathrm{eV}$ (HSE). Correspondingly, this structure has the higher gap both at the PBE or HSE levels. Finally, $16 \mathrm{c}$ is a $1 \mathrm{D}$ structure formed by the same four $(\mathrm{PbS})_{4}$ units. This structure is $0.19 \mathrm{eV}$ (both at the PBE and HSE levels) higher in energy than the planar structure $16 \mathrm{a}$.

The relative stabilities and gaps of $(\mathrm{PbS})_{32}$ structures deserve a separated comment as Kiran et al. have studied theoretically and by STM and XPS techniques $(\mathrm{PbS})_{32}$ clusters deposited in highly ordered porous graphite (HOPG) [11]. Many of the structures found by these authors in their STM images exhibit square or rectangular-like shapes. The apparition of those rectangular shape images was interpreted as resulting from the addition of two cubic $(\mathrm{PbS})_{32}$ units to form a larger $(\mathrm{PbS})_{64}$ cluster. This interpretation starts from the hypothesis that all $(\mathrm{PbS})_{32}$ units formed and initially deposited on the HOPG surface are cubic-shaped. However, our results allow us to offer a different interpretation as structures 32a (cubic shaped) and 32b (rectangular) have similar stabilities, while the rod-shaped 32c structure is always higher in energy by about $1 \mathrm{eV}$. While at the PBE level the cubic structure is more stable by $0.19 \mathrm{eV}$, at the HSE level both structures are degenerated (32b is more stable by only $0.01 \mathrm{eV}$ ). The inclusion of the SOC effects makes the rectangular 2D structure 32b to be more stable by $0.13 \mathrm{eV}$. Thus, the data of Kiran et al. can be interpreted as resulting from the initial formation and deposition of both 3D and 2D $(\mathrm{PbS})_{32}$ structures on the HOPG surface. Taking into account the low coverage employed in their experiments the interpretation that we offer here seems to be more plausible than the diffusion of on-surface $(\mathrm{PbS})_{32}$ units that join together to form larger $(\mathrm{PbS})_{64}$ units. The HOMO-LUMO gaps of both structures are quite similar, as expected from their similar relative energies. 
The observed dependence of the HOMO-LUMO gaps on the DFT functional employed and on the inclusion of the SOC effects implies that the electronic properties (i.e. the electronic absorption spectrum) will also be strongly dependent on these factors. In particular, Hummer et al. [16] have shown that in bulk lead chalcogenides standard DFT calculations (i.e. using either LDA or GGA approximations to treat the exchange correlation potential) results in negative band gaps and wrong order of band gaps. They also showed that a significant improvement could be obtained by the use of a hybrid functional and the inclusion of the SOC effects. The comparison of the computed electronic spectra of $(\mathrm{PbS})_{32}$ cubic structure obtained with $\mathrm{PBE}$ and HSE functionals and the inclusion of the SOC effects in the latter, presented in figure 5, confirm the importance of the use of a hybrid functional. While the onset of absorption starts at $\sim 2 \mathrm{eV}$ when the GGA functional is employed, the electronic absorption spectrum is displaced to higher energies by about $1 \mathrm{eV}$ when the HSE functional is used. The inclusion of the expensive SOC effects, in turn, displaces the spectrum to lower energies by $0.5 \mathrm{eV}$ but also changes its shape, presenting now a low energy tail that extends to $\sim 2.5 \mathrm{eV}$ the onset of absorption.

The evolution of the electronic absorption spectrum with NP size is presented on figure 6. These spectra show the expected evolution towards lower energies with increasing NP size. The most noteworthy feature is the higher energy absorption onset found on $(\mathrm{PbS})_{4}$ where the first intense maximum is found at $\sim 4.5 \mathrm{eV}$ while for the remaining NPs the comparable onset of absorption starts below $3 \mathrm{eV}$. In $(\mathrm{PbS})_{4}$ the DOS shows the LUMO to be found at $2.9 \mathrm{eV}$, the LUMO+1 at $3.9 \mathrm{eV}$ and the LUMO+2 at $4.4 \mathrm{eV}$. This means that transitions from the HOMO to LUMO or LUMO+1 are of quite low intensity and that the first clearly allowed transition is the HOMO $\rightarrow$ $\mathrm{LUMO}+2$. The spectra presented in figure 6 indicate that while $(\mathrm{PbS})_{32} \mathrm{NPs}$ present some absorption on the visible region of the spectrum (that extend from $1.65 \mathrm{eV}$ to $3.1 \mathrm{eV}$ ) larger NP sizes will be required to have intense visible and infrared absorption. We can estimate, from an extrapolation of the logarithmic fitting curve presented in figure 4, that to have a HOMO-LUMO gap, and thus absorption, in the IR/visible limit $(1.65 \mathrm{eV})$ a cubic NP of side length of more than $1.96 \mathrm{~nm}, \sim(\mathrm{PbS})_{250}$, should be necessary. This result is in agreement with experimental data that found that PbS NPs of diameter $2.6 \mathrm{~nm}$ have a gap of approximately $1.5 \mathrm{eV}$ [28]. 


\section{Conclusions.}

The structure of $(\mathrm{PbS})_{\mathrm{n}}$ nanoparticles with $\mathrm{n}=4-32$ is found to be quite different from bulk PbS. While in the latter all PbS bonds are equivalent, PbS NPs are found to be made by the successive addition of weakly interacting $(\mathrm{PbS})_{4}$ units. This results in each hexacoordinated $\mathrm{Pb}$ atom having two shorter, two intermediate, and two longer bond, a behaviour similar to that observed in molecular complexes with covalent $\mathrm{Pb}-\mathrm{S}$ bonds.

The distribution of bond distances has its reflection in the electronic structure. While there is a generic trend of HOMO-LUMO gap reduction with increasing NP size, the presence of longer and, thus, weaker bonds on some structures results in particularly low gaps. The choice of the functional used is found to strongly affect the computed electronic structure and, in particular, the inclusion of the SOC effects, while not having a significant effect on the bond distances, reduce the HOMO-LUMO gaps by $\sim 0.5 \mathrm{eV}$ and displace the electronic absorption spectra to lower energies. This confirms the need of accurate DFT calculations based on the HSE functional including the SOC effects to adequately reproduce the electronic structures of these systems. Finally, based on an extrapolation of our hybrid density functional calculations with SOC effects we predict that to have a HOMO-LUMO gap in the IR region of the spectrum $(1.65 \mathrm{eV})$ a cubic NP of at least $1.96 \mathrm{~nm}$ side length is required, a result which is in agreement with the experimental observation of band gap of $1.5 \mathrm{eV}$ for PbS QDs of $2.6 \mathrm{~nm}$ diameter.

Acknowledgements. This work was funded by the Junta de Andalucía, grant P12-FQM-1595 and EU FEDER. 


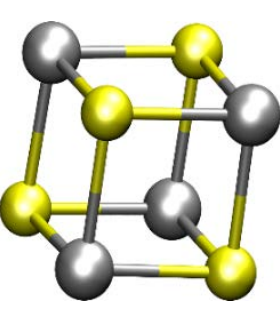

$4 a$

$\Delta \mathrm{E}=0.00 \mathrm{eV}$

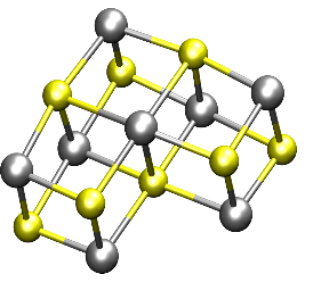

$8 a$

$\Delta E=0.49(0.58) \mathrm{eV}$

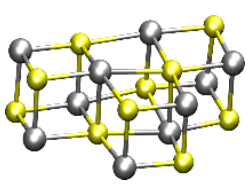

IOC

$\Delta \mathrm{E}=0.27(0.27) \mathrm{eV}$

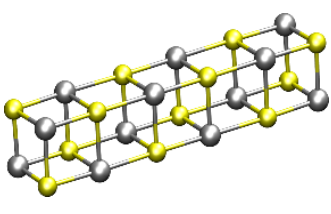

I2a

$\Delta \mathrm{E}=0.00 \mathrm{eV}$

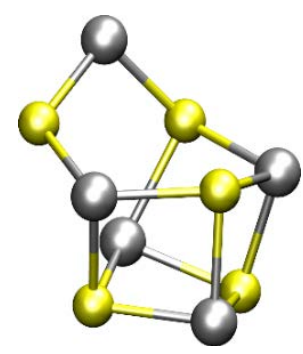

$5 a$

$\Delta \mathrm{E}=0.0 \mathrm{eV}$

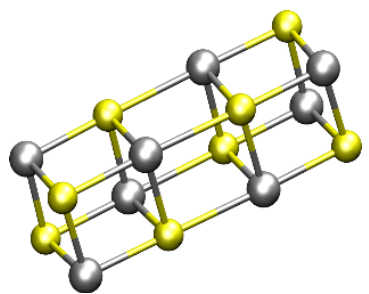

$8 b$

$\Delta \mathrm{E}=0.00 \mathrm{eV}$

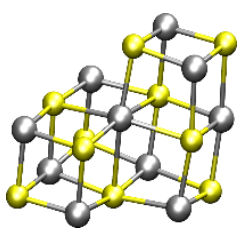

Iod

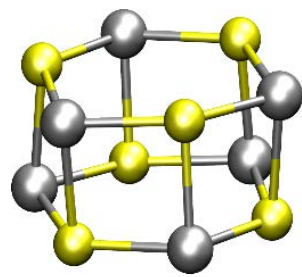

$6 a$

$\Delta E=0.09(0.15) e V$

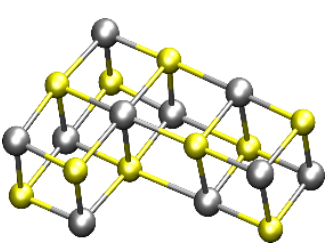

Ioa

$\Delta \mathrm{E}=0.27(0.30) \mathrm{eV}$

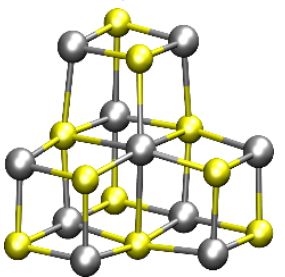

IOe

$\Delta E=0.9$ I $($ I.07) eV

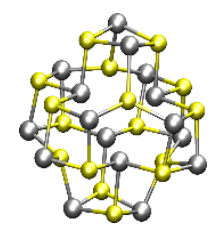

I6b

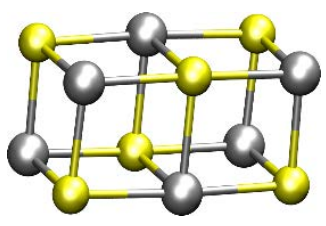

$6 b$

$\Delta \mathrm{E}=0.0 \mathrm{eV}$

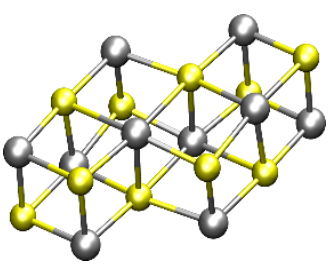

Iob

$\Delta E=0.50(0.57) \mathrm{eV}$

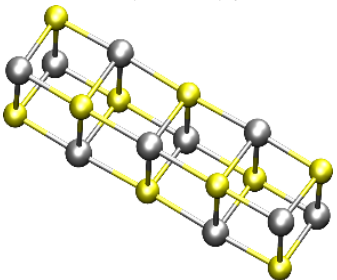

Iof

$\Delta \mathrm{E}=0.00 \mathrm{eV}$

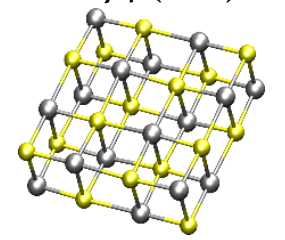

I6a

$\Delta \mathrm{E}=0.00 \mathrm{eV}$

$\Delta \mathrm{E}=0.6 \mathrm{I}(0.58) \mathrm{eV}$

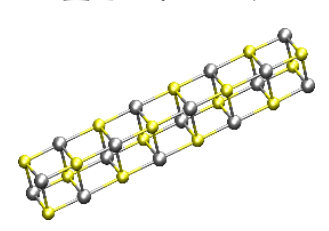

I6c

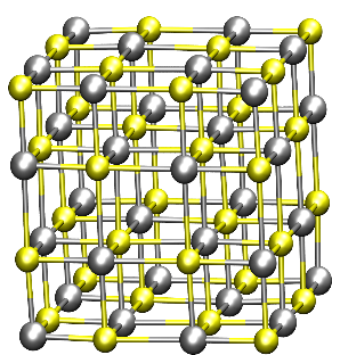

$32 \mathrm{a}$

$\Delta \mathrm{E}=0.00($ o.or $) \mathrm{eV}$

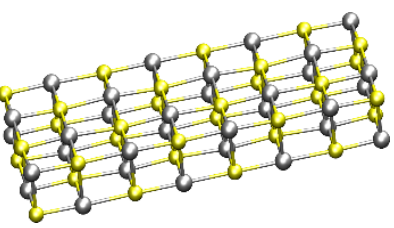

$32 \mathrm{~b}$

$\Delta \mathrm{E}=0.2 \mathrm{I}(0.00) \mathrm{eV}$

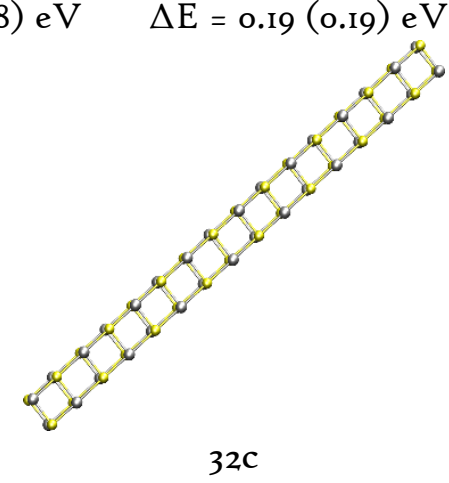

$\Delta \mathrm{E}=\mathrm{I} .24(\mathrm{I} .00) \mathrm{eV}$

Figure 1. Structures of $(\mathrm{PbS})_{\mathrm{n}}$ nanoparticles examined in the present paper and PBE (HSE) relative energies in $\mathrm{eV}$. 


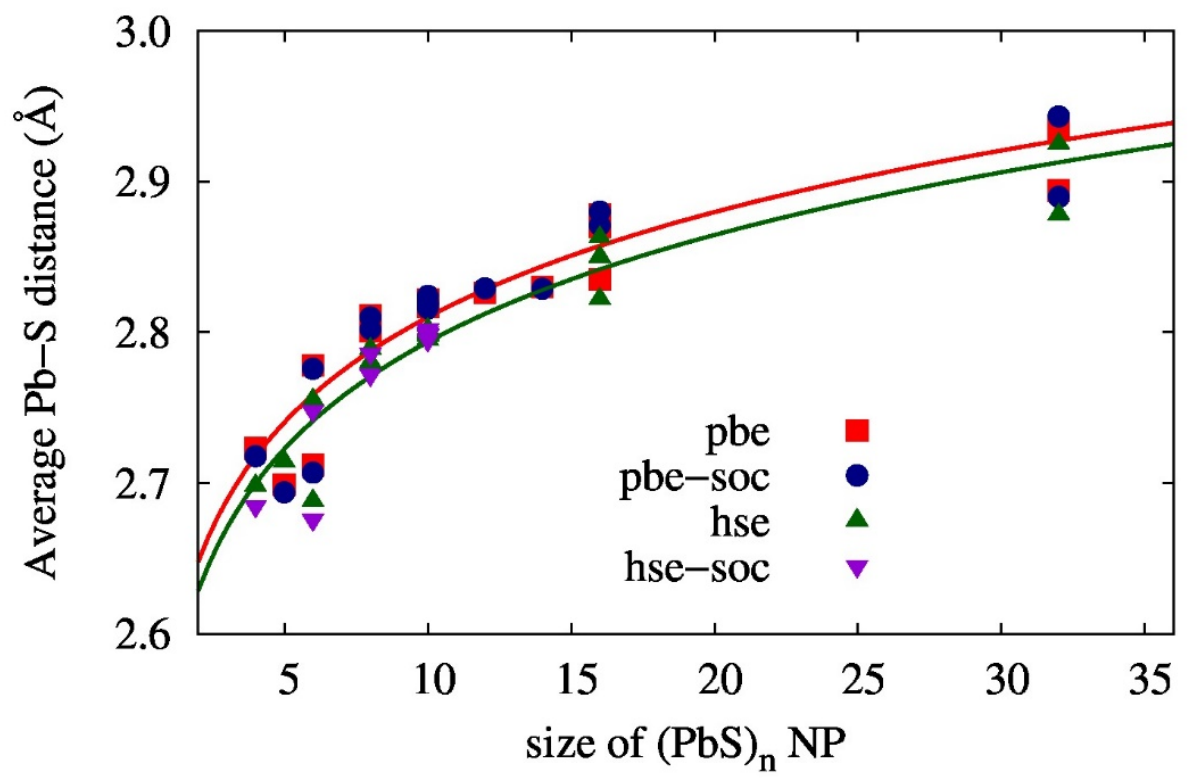

Figure 2. Calculated average Pb-S distances $(\AA)$ as a function of increasing NP size. 


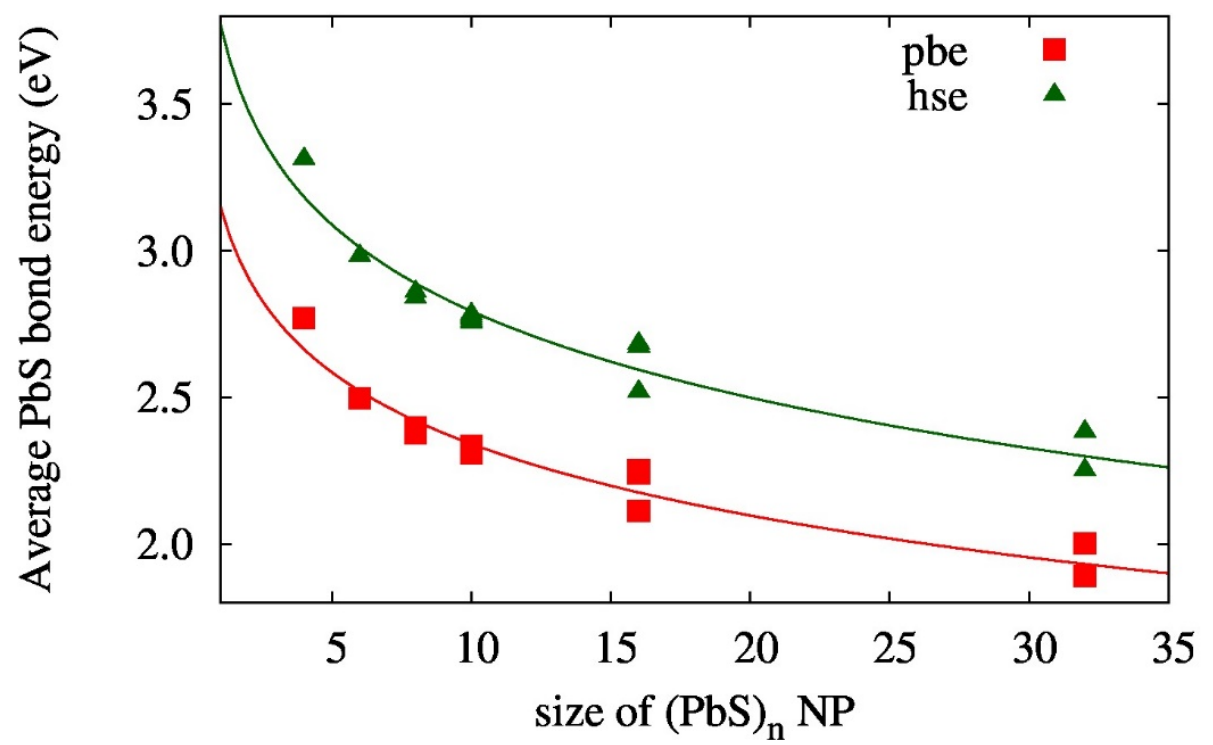

Figure 3. Computed average bond energy $(\mathrm{eV})$ as a function of increasing NP size. 


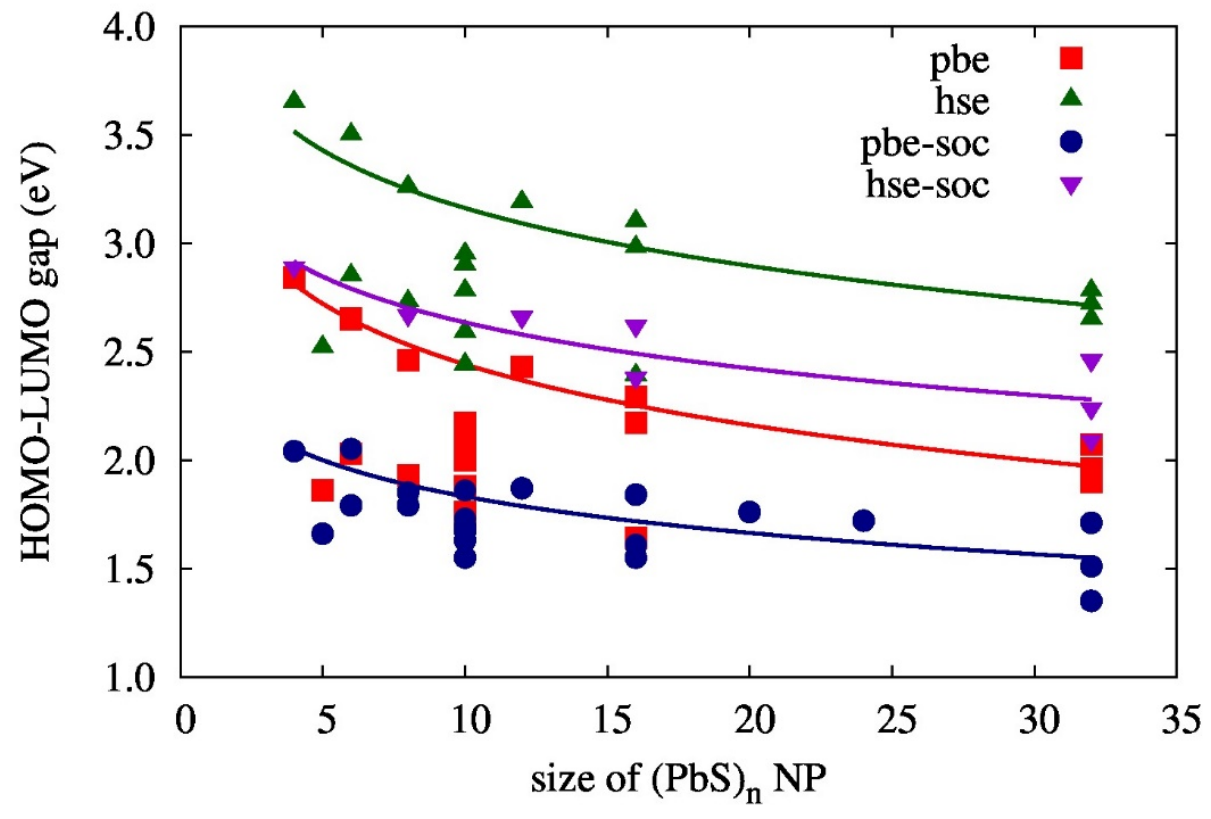

Figure 4. HOMO-LUMO (eV) gaps as a function of increasing NP size. 


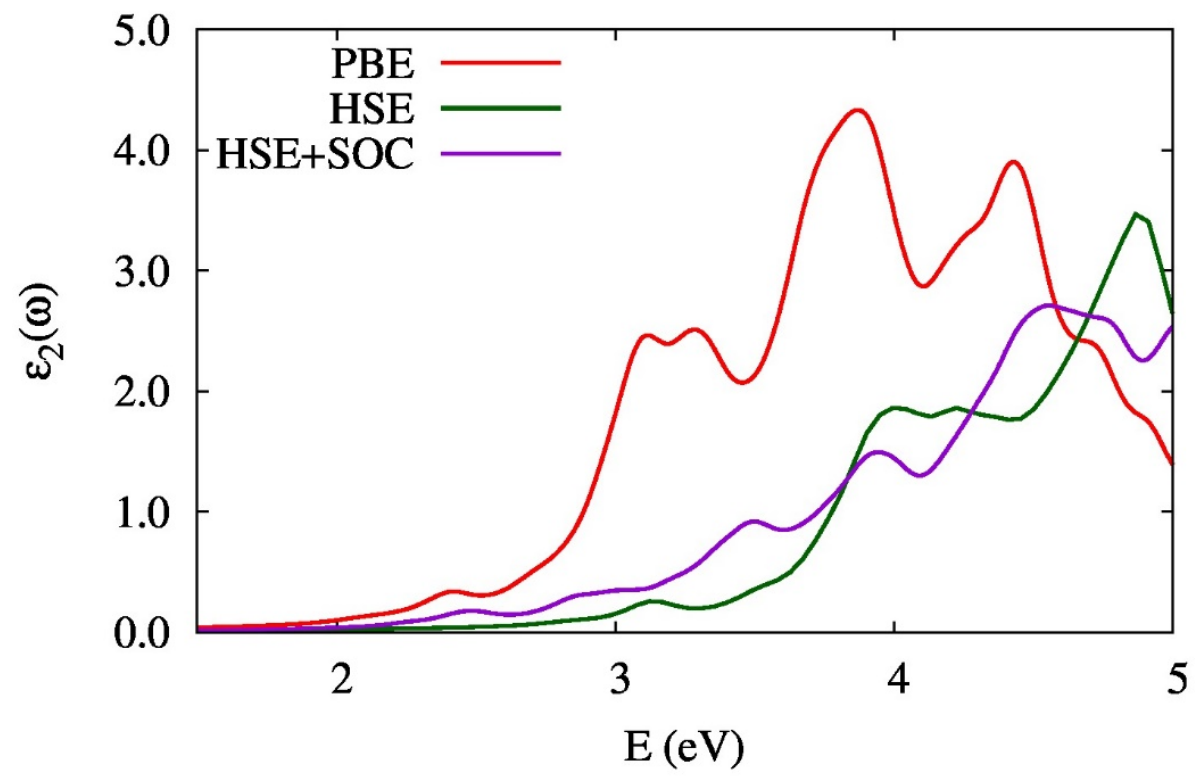

Figure 5. Computed electronic absorption spectra of $(\mathrm{PbS})_{32}$ cubic structure with different DFT functionals. 


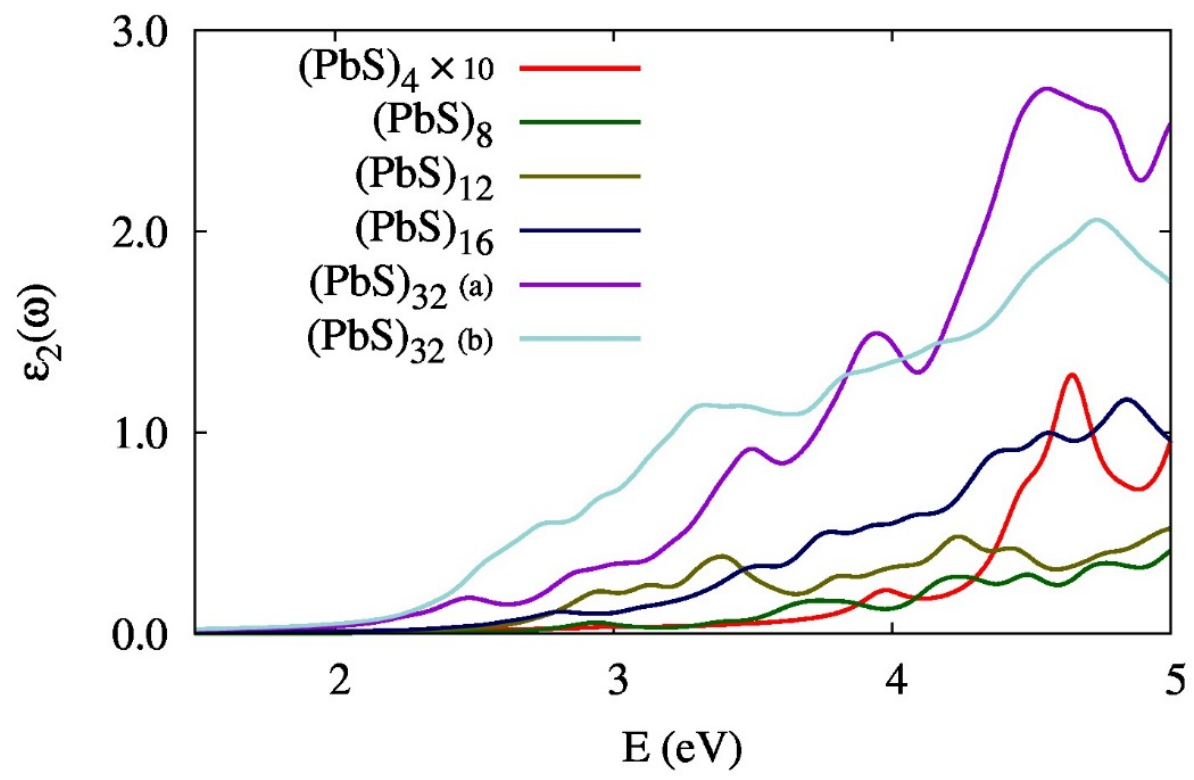

Figure 6. Computed electronic absorption spectra of different NPs at HSE+SOC level (all spectra normalized to the same cell volume). 


\section{References}

[1] Carey GH, Abdelhady AL, Ning Z, Thon SM, Bakr OM, Sargent EH (2015) Colloidal Quantum Dot Solar Cells. Chem Rev 115:12732-12763.

[2] Chen G, Seo J, Yang C, Prasad PN, (2013) Nanochemistry and Nanomaterials for Photovoltaics. Chem Soc Rev 42:8304-8338.

[3] Lee H, Leventis HC, Moon S-J, Chen P, Ito S, Haque SA, Torres T, Nüesch F, Geiger T, Zakeeruddin SM, Grätzel M, Nazeeruddin K (2009) PbS and CdS Quantum Dot-Sensitized Solid-State Solar Cells: “Old Concepts, New Results”. Adv Funct Mater 19:2735-2742.

[4] Talapin DV, Lee J-S, Kovalenko MV, Shevchenko EV (2010) Prospects of Colloidal Nanocrystals for Electronic and Optoelectronic Nanomaterials for Photovoltaics. Chem Rev 110:389-458.

[5] Zogg H, Maissen C, Masek J, Hoshino T, Blunier S, Tiwari AN (1991) Photovoltaic infrared sensor arrays in monolithic lead chalcogenides on silicon. Semicond Sci Technolog 6:C36-C41. [6] Liu S, Tang Z (2010) Nanoparticle Assemblies for Biological and Chemical Sensing. J Mater Chem 20:24-35.

[7] Qiao L-N, Wang HC, Shen Y, Lin Y-H, Nan C-W (2016) Visible-active photocatalytic behaviors observed in nanostructured lead chalcogenides $\mathrm{PbX}(\mathrm{X}=\mathrm{S}$, Se, Te). AIP Advances, 6:015108.

[8] Hines MA, Scholes GD (2003) Colloidal PbS Nanocrystals with Size-Tunable Near-Infrared Emission: Observation of Post-Synthesis Self-Narrowing of the Particle Size Distribution. Adv Mater 15:1844-1849.

[9] Moreels I, Justo Y, Geyter BD, Haustraete K, Martins JC, Hens Z (2011) Size-Tunable, Bright, and Stable PbS Quantum Dots: A Surface Chemistry Study. ACS Nano 5:2004-2012.

[10] Sykora M, Koposov AY, McGuire JA, Schultze RK, Tretiak O, Pietryga JM, Klimov VI (2010) Effect of Air Exposure on Surface Properties, Electronic Structure, and Carrier Relaxation in PbSe Nanocrystals. ACS Nano 4:2021-2034.

[11] Kiran B, Kandalam AK, Rallabandi R, Koirala P, Li X, Tang X, Wang Y, Fairbrother H, Gantefoer G, Bowen K (2012) (PbS) $)_{32}$ : A baby crystal. J Chem Phys 136:024317.

[12] Zeng H, Schelly ZA, Ueno-Noto K, Marynick DS (2005) Density Functional Study of Lead Sulfide Clusters (PbS) $(\mathrm{n}=1-9)$. J Chem Phys A 109:1616-1620.

[13] He J, Liu C, Li F, Sa R, Wu K (2008) Size-dependence of stability and optical properties of lead sulfide clusters. Chem Phys Lett 457:163-168.

[14] Graciani J, Márquez AM, Plata JJ, Ortega Y, Hernández NC, Meyer A, Zicovich-Wilson CM, Fdez Sanz J (2011) Comparative Study of the Performance of Hybrid DFT Functionals in Highly Correlated Oxides: The Case of $\mathrm{CeO}_{2}$ and $\mathrm{Ce}_{2} \mathrm{O}_{3}$. J Chem Theor Comput 7:56-65. 
[15] Paier J, Marsman M, Hummer K, Kresse G, Gerberm IC, Ángyán JC (2006) Screened hybrid density functionals applied to solids. J Chem Phys 124:154709.

[16] Hummer K, Grüneis A, Kresse G (2007) Structural and electronic properties of lead chalcogenides from first principles. Phys Rev 75:195211.

[17] Kresse G, Furthmuller J (1996) Efficient iterative schemes for ab initio total-energy calculations using a plane-wave basis set. Phys Rev B 54:11169-11186.

[18] Kresse G, Furthmuller J (1996) Efficiency of ab-initio total energy calculations for metals a semiconductors using a plane-wave basis set. Comput Mater Sci 6:15-50.

[19] Kresse G, Hafner J (1993) Ab initio molecular dynamics for liquid metals. Phys Rev B 47:558-561.

[20] Kresse G, Joubert D (1999) Fast ultrasoft pseudopotentials to the projector augmentedwave method. Phys Rev B 59:1758-1775.

[21] Blöch PE (1994) Projector augmented-wave method. Phys Rev B 50:17953-17979.

[22] Perdew JP, Burke K, Ernzerho, M (1996) Generalized Gradient Approximation Made Simple. Phys Rev Lett 77:3865-3868.

[23] Krukau AV, Vydrov OA, Izmaylov AF, Scuseria GE (2006) Influence of the exchange screening parameter on the performance of screened hybrid functionals. J Chem Phys 125:224106.

[24] Harris J (1985) Simplified method for calculating the energy of weakly interacting fragments. Phys Rev B 31:1770-1779.

[25] Afzaal M, Ellwood K, Pickett NL, O’Brien P, Raftery J, Waters J (2004) Growth of lead chalcogenide thin films using single-source precursors. J Mater Chem 14:1310-1315.

[26] Iwasaki H, Hagihara H (1972) The Cristal Structure of Lead (II) Diethyldithiocarbamate. Acta Crystallogr Sect. B 28:507-513.

[27] Franceschetti A (2008) Structural and electronic properties of PbSe nanocrystals from first principles. Phys Rev B 78:075418.

[28] Choi H, Ko J-H, Kim Y-H, Jeong S (2013) Steric-Hindrace-Driven Shape Transition in PbS Quantum Dots: Understanding Size-Dependent Stability. J Am Chem Soc 135:5278-5281. 